CLINICAL STUDY

\title{
Serological screening for coeliac disease in adults with Turner's syndrome: prevalence and clinical significance of endomysium antibody positivity
}

\author{
Amy R Frost, Margaret M Band and Gerard S Conway \\ Department of Endocrinology, University College Hospital, 250 Euston Road, London NW1 2QP, UK \\ (Correspondence should be addressed to G S Conway; Email: gerard.conway@uclh.nhs.uk)
}

\begin{abstract}
Objective: To investigate the prevalence of coeliac disease (CD) in an adult population with Turner's syndrome (TS).

Design: A clinic population with TS was screened using a serological test for CD.

Methods: Two hundred and fifty six patients with TS were included in the study. Five patients had existing diagnoses of $\mathrm{CD}$. The remaining 251 asymptomatic patients were screened using an IgA endomysium antibody (EMA) test. Positive cases were offered endoscopy with duodenal biopsy. HLA typing was undertaken in existing cases and new EMA-positive cases.

Results: Of the 251 patients screened, eight were found to be EMA positive (3.2\%). Seven patients proceeded to duodenal biopsy on which all were confirmed histologically to have cluster of differentiation $(2.8 \%)$. The prevalence of subclinical cluster of differentiation in the population can therefore be estimated between 2.8 and $3.2 \%$. The total population prevalence of CD, including the previously diagnosed cases, is estimated between 4.7 and $5.1 \%$. Ten patients with histologically confirmed cluster of differentiation underwent HLA typing of which eight were HLA-DQ2 positive, one was HLA-DQ8 positive and one was negative to both HLA-DQ2 and HLA-DQ8.

Conclusions: This study demonstrates an increased prevalence of cluster of differentiation in an adult population with TS over the general population. This is consistent with previous data published in paediatric populations.
\end{abstract}

European Journal of Endocrinology 160 675-679

\section{Introduction}

The hallmark of Turner's syndrome (TS) is an absent or structurally abnormal X- chromosome in a phenotypic female. It has long been recognized that there is an increased incidence of autoimmune disorders in TS (1), with hypothyroidism being the most common. Many studies have reported an increased prevalence of coeliac disease (CD) in TS (2-9), with prevalences reported of $0-8.1 \%$, compared with $0.5-1.0 \%$ in the general population, based on serological screening (10).

Coeliac screening studies in TS have thus far been performed in paediatric populations, and we note that the risk of developing autoimmune thyroid disease increases with age (11). We have therefore undertaken serological screening for cluster of differentiation in an adult population with TS to determine the degree of age progression of this condition.

Recent advances in understanding the pathogenesis of cluster of differentiation have pointed to the modification of wheat gluten proteins by tissue transglutaminase (12), with a key role of HLA-DQ2 in presenting toxic wheat proteins to $\mathrm{T}$ cells. Tissue transglutaminase is the target of the antiendomysial autoantibody response in CD. Antibodies to endomysium (IgA endomysium antibody, EMA) can be measured in serum via indirect immunofluoresence to provide a serological test for cluster of differentiation which has been shown to be highly sensitive and specific, with values for both parameters exceeding 95\% in most studies (13). An alternative approach using an enzyme linked immunoabsorbent assay measuring IgA tissue transglutaminase antibody (tTGA) levels has comparable sensitivity and specificity, however, incomplete concordance between the two tests has led some authors to suggest combination screening (14). By contrast, the previously widely used antigliadin tests have been shown to have relatively poor sensitivity and specificity.

While serological screening remains a powerful tool in the diagnosis of $\mathrm{CD}$, it has been demonstrated that EMA sensitivity is reduced in cases of partial rather than total villous atrophy (15), and the gold standard for the diagnosis of cluster of differentiation remains the finding of villous atrophy on duodenal biopsy. 


\section{Subjects and methods}

Two hundred and fifty six consecutive women with karyotypically proven TS attending the Adult Turner clinic at University College Hospital, London were screened as a routine part of their health surveillance programme. Ethical committee approval was given for clinical notes review.

All subjects were screened using a serological test for EMA positivity. Indirect immunofluorescence analysis was performed using commercially available fixed sections of monkey oesophagus (Biodiagnostics Ltd, Worcestershire, UK) as antigen substrate. Patient serum was diluted 1:10 with PBS. Patient endomysium specific IgA was detected with a FITC-labelled sheep antihuman IgA conjugate (Dako Ltd, Ely, UK F0204). All positive patients were offered a duodenal biopsy. These were undertaken either in our centre or at the patient's local centre, in accordance with routine practice. We accepted routine pathology reports as evidence of cluster of differentiation as we were unable to obtain original slides from all centres to enable a uniform reporting system.

HLA typing was offered to all patients with positive EMA serology or a previous diagnosis of CD. DNA was extracted from EDTA blood using a Qiagen MagAttract DNA Cell Mini Kit by a M48 BioRobot and analysed using the Protrans Domino System HLA CD Association kit.

\section{Results}

The median age of the 256 patients with TS was 29 (16-61) years. Five patients had an established diagnosis of cluster of differentiation made prior to the transition to adult care, following a variety of clinical presentations. In order to clarify the effect of ascertainment bias affecting the screening statistics, we have presented prevalence data for cluster of differentiation with and without these cases included. The characteristics of these five cases are presented with those of the newly identified EMA-positive cases (Table 1). The 251 remaining patients with TS were screened for EMA positivity.

EMA were positive in $8 / 251$ (3.2\%) patients, none of whom reported symptoms suggestive of CD. Duodenal biopsy was declined by one patient; the remaining seven proceeded to biopsy that confirmed the diagnosis of cluster of differentiation histologically, with partial or total villous atrophy in all cases $(7 / 251$ or $2.8 \%)$. If the five previously diagnosed cases of cluster of differentiation were included then the total population prevalence of cluster of differentiation would be $12 / 256(4.7 \%)$.

HLA typing was performed in 10 women with histologically confirmed cluster of differentiation (six newly diagnosed and four previously diagnosed); eight were HLA-DQ2 positive, one was HLA-DQ8 positive and one was negative for both HLA-DQ2 and HLA-DQ8.

In order to place our findings in a wider perspective, we combined our data with that from all published studies found in a search of Medline (Table 2). The prevalence of EMA positivity detected by EMA/tTGA screening was $27 / 638(4.2 \%)$. Of the 21 EMA-positive cases who proceeded to biopsy, $18(85.7 \%)$ had histological confirmation of $\mathrm{CD}$, with five studies showing $100 \%$ concordance between EMA positivity and biopsy-proven $\mathrm{CD}$.

\section{Discussion}

This study confirms the finding of an increased prevalence of cluster of differentiation in TS and, interestingly, the prevalence in an adult population is similar to that reported in paediatric populations (Table 2) (2-9). Combining all studies, the prevalence of screening-detected EMA positivity in TS is $4.2 \%$, which represents a 4 to 8 fold increased risk over the general population. The fact that there appears to be little age-related progression in the prevalence of cluster of differentiation is in contrast to the natural history of hypothyroidism in adults with TS (11) and raises the possibility that a single screening exercise will ascertain most cases. It might be prudent to undertake this during paediatric care, and certainly before treatment with growth hormone is considered, as untreated CD could impact on growth velocity. Reviewing the data in the TS population, serological screening appears to be an effective method of identifying subclinical $\mathrm{CD}$. While HLA screening is a possible alternative, in our population, one HLA-DQ2/8 negative case was identified which raises the possibility of false negatives in the TS population.

It is interesting to speculate on the mechanism for this increased prevalence of cluster of differentiation and other autoimmune diseases in TS. Cluster of differentiation is a strongly heritable condition, with a $75 \%$ concordance in monozygotic twins compared with $11 \%$ in dizygotic twins (16). More than $90 \%$ of patients diagnosed with cluster of differentiation have the HLADQ2 haplotype, with virtually all the rest having HLADQ8 (17). This compares with a European population prevalence of these HLA subtypes of between 20 and $30 \%$ (18). There appears to be no increased representation of these genotypes in TS; in the one TS population that has been studied to date, $14 / 46(29.1 \%)$ were found to be HLA-DQ2 positive, comparable with the general population (6). From these observations, we conclude that a 'second hit' of autoimmune activation of this HLA risk group must be behind the excess of risk of cluster of differentiation in TS. Of note, there was no significant relationship between hypothyroidism (6/13 women with $C D)$ and cluster of differentiation in this audit. 


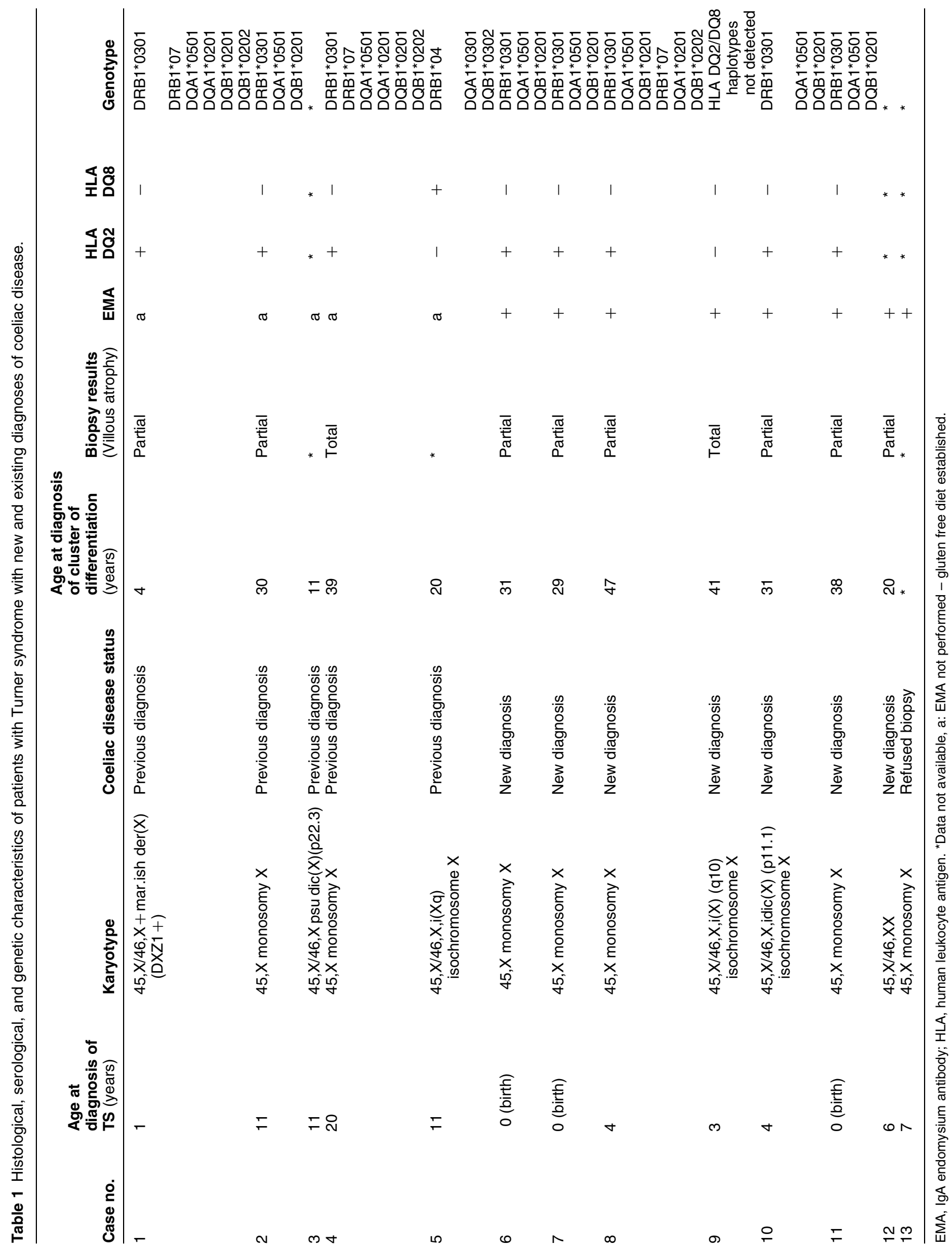




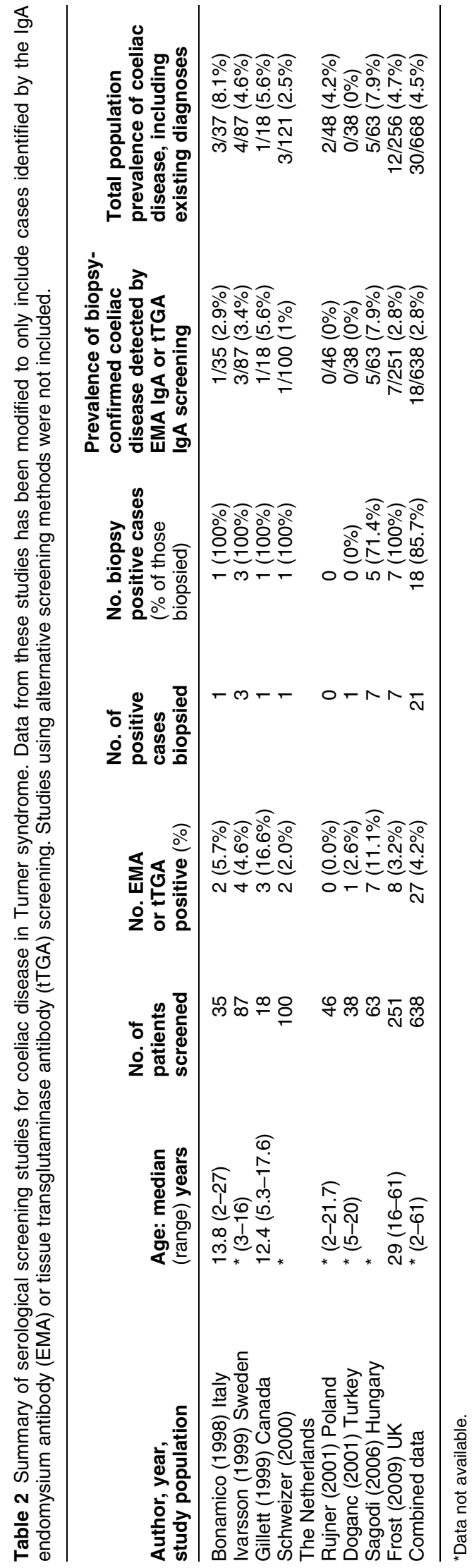

All eight previously reported cases of TS and cluster of differentiation that have been subjected to HLA typing have been found to carry HLA-DQ2 $(2,5,6)$. Interestingly, one subject ( $1 / 8$ or $12.5 \%$ ) in our study population with positive EMA serology and histologically confirmed CD was HLA-DQ2 and DQ8 negative. This is rare in the general population with cluster of differentiation and is the first case to be reported in the Turner's population. In a large European study of 1008 patients with CD, only 61 (6.1\%) HLA-DQ2/DQ8 negative cases were described (17). Of these 61 cases, 57 encoded half of the HLA DQ2 heterodimer. It has been suggested that the remaining four cases could carry rare mutations in other HLA-DQ alleles, enabling them to present gliadin to T cells (18). Although only one case of HLA-DQ2/DQ8 negative cluster of differentiation was identified here, it is nevertheless tempting to speculate that this case adds further weight to the notion of an exaggerated 'second hit' in the development of cluster of differentiation in TS. TS therefore provides an important model for the study of the origin of autoimmune disorders, with X-chromosome genes and oestrogen deficiency as possible mechanisms.

When considering screening for subclinical cluster of differentiation in TS, it is important to assess what the clinical significance of the diagnosis may be in this population. Some studies suggest that people with undetected cluster of differentiation have a tendency towards low bone density $(19,20)$ and mild nutritional deficits (21). Even in our large study, our population with TS and cluster of differentiation was too small to provide sufficient power to address this issue with statistical significance.

There is, however, one scenario when it would be particularly prudent to undertake cluster of differentiation screening and that is in the pre-pregnancy work-up of women with TS seeking ovum donation. Undiagnosed cluster of differentiation at the time of delivery may be associated with pre-term birth, intrauterine growth retardation and low or very low birth weight (22), all of which are already prevalent in TS pregnancies (23). This is in contrast to maternal cluster of differentiation diagnosed before birth which is not associated with adverse foetal outcomes.

In conclusion, our findings confirm an increased prevalence of cluster of differentiation in TS, estimated in our population as lying between $4.7 \%$ (biopsy confirmed) and 5.1\% (EMA positive). When all studies are combined, a total population prevalence of cluster of differentiation in TS is estimated at $4.5 \%$. The potential advantages to the detection of clinically silent cluster of differentiation in this population, such as beneficial effects on bone mineral density, nutritional status, and pregnancy outcomes remain to be clarified.

\section{Declaration of interest}

There is no conflict of interest that could be perceived as prejudicing the impartiality of the research reported. 


\section{Funding}

This research did not receive any specific grant from any funding agency in the public, commercial or not-for-profit sector.

\section{Author contribution statement}

ARF, data collection, manuscript writing; MMB, coordination of HLA typing; GSC, concept, revision of manuscript.

\section{Acknowledgements}

This work was undertaken at UCLH/UCL who received a proportion of funding from the Department of Health's NIHR Biomedical Research Centres funding scheme.

\section{References}

1 Fialkow PJ. Autoimmunity and chromosomal aberrations. American Journal of Human Genetics 196618 93-108.

2 Bonamico M, Bottaro G, Pasquino AM, Caruso-Nicoletti M, Mariani P, Gemme G, Paradiso E, Ragusa MC \& Spina M. Celiac disease and Turner syndrome. Journal of Pediatric Gastroenterology and Nutrition 199826 496-499.

3 Ivarsson S-A, Carlsson A, Bredberg A, Alm J, Aronsson S, Gustafsson J, Hagenas L, Hager A, Kristrom B, Marcus C, Moell C, Nilsson KO, Tuvemo T, Westphal O, AlbertssonWikland K \& Aman J. Prevalence of coeliac disease in Turner syndrome. Acta Paediatrica 199988 933-936.

4 Gillett PM, Gillett HR, Israel DM, Stewart L, Metzger DL, Chanoine J-P \& Freeman HJ. Increased prevalence of celiac disease in girls with Turner syndrome. Journal of Pediatric Gastroenterology and Nutrition 199929497.

5 Schweizer JJ, Mearin ML, Brand R \& Wit J-M. Increased frequency of celiac disease in Turner syndrome in the Netherlands. Journal of Pediatric Gastroenterology and Nutrition 200031 S144-S145.

6 Rujner J, Wisniewski A, Grogorek H, Wozniewicz B, Mlynarski W \& Witas HW. Coeliac disease and HLA-DQ2 (DQA1*0501 and DQB1*0201) in patients with Turner syndrome. Journal of Pediatric Gastroenterology and Nutrition 200132 114-115.

7 Doganc T, Caliskan L \& Vidinlisan S. Celiac disease and HLA-DQ2 association in patients with Turner syndrome. Journal of Pediatric Gastroenterology and Nutrition 200133352.

8 Bonamico M, Pasquino AM, Mariani P, Danesi HM, Cutlasso F, Mazzanti L, Petri A \& Bona G. Prevalence and clinical picture of celiac disease in Turner syndrome. Journal of Clinical Endocrinology and Metabolism 200287 5495-5498.

9 Sagodi L, Solyam E, Tamasi K \& Minik K. Prevalence of coeliac disease in Turner syndrome. Orvosi Hetilap 2006 147 1185-1188.

10 Van Heel DA \& West J. Recent advances in coeliac disease. Gut 200655 1037-1046.

11 Elsheikh M, Wass JAH \& Conway GS. Autoimmune thyroid syndrome in women with Turner's syndrome - the association with karyotype. Clinical Endocrinology 200155 223-226.
12 Molberg O, Mcadam SM, Korner R, Quarsten H, Kristiansen C, Madsen L, Fugger L, Scott H, Noren O, Roepstorff P, Lundin KE, Sjostrom H \& Sollid LM. Tissue transglutaminase selectively modifies gliadin peptides that are recognized by gut-derived T cells in celiac disease. Nature Medicine 19984 713-717.

13 Hill ID. What are the sensitivity and specificity of serologic tests for celiac disease? Do sensitivity and specificity vary in different populations? Gastroenterology 2005128 S25-S32.

14 Dickey W, McMillan SA \& Hughes DF. Sensitivity of serum tissue transglutaminase antibodies for endomysial antibody positive and negative coeliac disease. Scandinavian Journal of Gastroenterology 200136 511-514.

15 Rostami K, Kerchhaert J, Tiemessen R, von Blomberg BM, Meijer JW \& Mulder CJ. Sensitivity of antiendomysium and antigliadin antibodies in untreated celiac disease: disappointing in clinical practice. American Journal of Gastroenterology $1999 \mathbf{9 4}$ 888-894.

16 Greco L, Romino R, Coto I, Di Cosmo N, Percopo S, Maglio M, Paparo F, Gasperi V, Limongelli MG, Cotichini R, D’Agate C, Tinto N, Sacchetti L, Tosi R \& Stazi MA. The first large population based twin study of coeliac disease. Gut $2002 \mathbf{5 0} 624-628$.

17 Karell K, Louka AS, Moodie SJ, Ascher H, Clot F, Greco L, Ciclitera PJ, Sollid LM \& Partanen J. HLA types in celiac disease patients not carrying the DQA $1 * 05-\mathrm{DQB} 1{ }^{*} 02(\mathrm{DQ} 2)$ heterodimer: results from the European Genetics Cluster on Celiac Disease. Human Immunology 200364 469-477.

18 Kipatany A, Toth L, Tumpek J, Csipo I, Sipos E, Woolley N, Partanen J, Szegedi G, Olah E, Sipka S \& Korponay-Szab IR. Diagnostic significance of HLA-DQ typing in patients with previous coeliac disease diagnosis based on histology alone. Alimentary Pharmacology \& Therapeutics 200624 1395-1402.

19 Corazza GR, Di Sario A, Cecchetti L, Jorizzo RA, Di Stefano M, Minguzzi L, Brusco G, Bernardi M \& Gasbarrini G. Influence of pattern of clinical presentation and of gluten-free diet on bone mass and metabolism in adult coeliac disease. Bone 1996 18 525-530.

20 Mustalahti K, Collin P, Sievanen H, Salmi J \& Maki M. Osteopenia in patients with clinically silent coeliac disease warrants screening. Lancet $1999 \mathbf{3 5 4} 744-745$.

21 Haapalahti M, Kulmala P, Karttunen TJ, Paajanen L, Laurila K, Maki M, Mykkanen H \& Kokkonen J. Nutritional status in adolescents and young adults with screen-detected celiac disease. Journal of Pediatric Gastroenterology and Nutrition $2005 \mathbf{4 0}$ $566-570$.

22 Ludvigsson JF, Montgomery SM \& Ekbom A. Celiac disease and risk of adverse fetal outcome: a population-based cohort study. Gastroenterology 2005129 454-463.

23 Abir R, Fisch B, Nahum R, Orvieto R, Nitke S \& Ben Rafael Z. Turner's syndrome and fertility: current status and possible putative prospects. Human Reproduction Update $2001 \quad 7$ 603-610.

Received 26 January 2009

Accepted 3 February 2009 